\title{
Randomised Trial of Lipiodol Uterine Bathing Effect (LUBE) in Women with Endometriosis-Related Infertility
}

\author{
N.P. Johnson ${ }^{1,2,3, \star}$, S. Baidya ${ }^{2}$, S.O. Jessup ${ }^{4}$, C.G. Print ${ }^{5}$, A. Muthukaruppan ${ }^{2}$, L.W. Chamley ${ }^{2}$, \\ W.E. Hadden ${ }^{6}$, M.L. Hull ${ }^{1}$, S. Mehta ${ }^{5}$, A.N. Shelling ${ }^{2}$ \\ ${ }^{1}$ Robinson Research Institute, University of Adelaide, Australia \\ ${ }^{2}$ Department of Obstetrics and Gynaecology, The University of Auckland, New Zealand \\ ${ }^{3}$ Repromed Auckland; Auckland Gynaecology Group, 105 Remuera Road, Auckland, New Zealand \\ ${ }^{4}$ Demeter Fertility Clinic, Sydney, New South Wales, Australia \\ ${ }^{5}$ Department of Molecular Medicine and Pathology; Maurice Wilkins Centre; \\ The University of Auckland, New Zealand \\ ${ }^{6}$ Auckland Radiology Group, 101 Remuera Road, Auckland, New Zealand
}

\begin{abstract}
BACKGROUND: We aimed to assess whether lipiodol alters endometrial gene expression through a uterine bathing effect that might enhance receptivity to embryo implantation.

METHODS: An open-label randomised controlled trial design in a single-centre tertiary infertility service. Twelve women with endometriosis $(n=11)$ or previous successful lipiodol procedure $(n=1)$ were randomised to receive immediate or delayed lipiodol hysterosalpingography, followed by endometrial biopsy. Endometrial samples were assessed for gene expression, using Affymetrix microarrays and validation studies using reverse transcriptase quantitative polymerase chain reaction analysis. Subsequent endometrial gene expression responses to treatment and clinical fertility outcomes were assessed.

RESULTS: Eleven of 12 women had successful endometrial sampling procedures. Nine women had successful pregnancies within the 9-month follow-up phase. Following lipiodol bathing we identified 20 down-regulated and 13 up-regulated genes with $p \leq 0.05$ and with magnitude of change $\geq 1.5$-fold in at least three of the four women, with osteopontin being the only gene down-regulated in all four women.

CONCLUSIONS: This study supports the concept of a uterine bathing effect of lipiodol altering endometrial biology and gene expression. Whether regulation of inflammation and immune response pathways by lipiodol might contribute to an increase in endometrial receptivity to embryo implantation merits further investigation.
\end{abstract}

Keywords: Endometriosis; Lipiodol; Microarray; Osteopontin; Randomised Controlled Trial; Uterine Bathing.

\section{INTRODUCTION}

Fertility benefit has been demonstrated from a hysterosalpingogram (HSG) with oil soluble contrast media (OSCM) such as lipiodol versus no intervention (Mohiyiddeen et al., 2015) and now versus water soluble contrast media (WSCM) in a Dutch multi-centre trial that has re-invigorated interest in the fertility benefit of lipiodol (Dreyer et al., 2017). We found the most marked benefit amongst women with endometriosis (Johnson et al., 2004). We have shown that lipiodol does not need to flush the fallopian tubes to attain this benefit (Johnson, 2014) and we have proposed a possible uterine bathing effect of lipiodol that might alter the endometrial environment thus enhancing implantation (Johnson et al., 2005).
Our aim was to test the hypothesis that lipiodol regulates endometrial gene expression through a uterine bathing effect that might enhance receptivity to embryo implantation.

\section{MATERIALS AND METHODS}

\section{Ethics and consent}

A single-centre open-label parallel group randomised controlled trial (RCT) was undertaken in women with a good prognosis for treatment success following lipiodol uterine bathing treatment (i.e., who either had endometriosis or a previous successful pregnancy following lipiodol). Approval for the trial was granted by the New Zealand Health and Disability National Ethics Committee (approval number AKY/04/06/144) prior to commencement and annually

() 2019 by the Asia Pacific Initiative on Reproduction (ASPIRE) and World Scientific Publishing Co. Pte. Ltd. 2 Open Access article under the CC BY-NC-ND license (http://creativecommons.org/licenses/by-nc-nd/4.0/).

Received 1 February 2019; Accepted 18 March 2019; Published 29 March 2019

${ }^{*}$ Correspondence should be addressed to: Dr. Neil Johnson, Repromed Auckland; Auckland Gynaecology Group, 105 Remuera Road, Auckland, New Zealand. Email: neiljohnson1964@gmail.com 
thereafter; the ethical standards of the Helsinki Declaration of 1975, as revised in 1983, were met. This RCT was not registered a priori in a clinical trial registry as recruitment took place from 2005 to 2006 before this requirement was in place. The full protocol for the trial has previously been described (https://researchspace.auckland. ac.nz/bitstream/handle/2292/5544/02 whole.pdf? sequence=2) (Johnson, 2007). All study participants gave full informed signed consent to participate in the research.

\section{Study design}

Women were randomised to receive lipiodol either 'immediately' (on the same cycle as randomisation) or 'after delay' of at least three completed menstrual cycles. Endometrial sampling procedures were undertaken, then fertility outcomes were then assessed. Women were asked to refrain from other fertility treatments from randomisation for the 9-month duration of follow-up. The design of the study was such that a comparison could be made between: (a) women randomised to immediate lipiodol versus those not randomised to immediate lipiodol, (b) women subsequently becoming pregnant versus those not subsequently becoming pregnant, (c) pre- and postlipiodol amongst individual women (Fig. 1). No formal sample size calculation was undertaken, but we estimated that approximately 12 participants would be required to show important differences in endometrial gene activation between women receiving lipiodol versus those not receiving lipiodol.

Eligibility criteria were women aged 18 to 39 years inclusive, with infertility of duration 12 months or more, who either had endometriosis (confirmed previously through laparoscopic inspection by a gynaecologist, but if laparosopic removal of endometriosis had been undertaken, this was more than 6 months previously) or who had previously become pregnant within 6 months of a lipiodol bathing procedure. Full investigation of infertility needed to have confirmed bilateral tubal patency either by dye studies at laparoscopy or hysterosalpingogram (HSG), early follicular phase follicle stimulating hormone (FSH) level of $10 \mathrm{IU} / \mathrm{L}$ or less; mid-luteal progesterone level of $25 \mathrm{mmol} / \mathrm{L}$ or more in a spontaneous cycle; the partner's semen analysis had to be normal by the existing World Health Organization (1992) criteria. Exclusion criteria included tubal damage, previous ectopic pregnancy, or iodine allergy.

Women were recruited from a tertiary level fertility clinic in Auckland, New Zealand (Fertility Plus, Auckland District Health Board). Women were randomised at the beginning of menses. Randomisation was performed using a computer-generated random number sequence (unknown to the executors of the assignment). The randomisation was in a block of 12 but this was unknown to all, other than a statistician who generated the allocation sequence and the principal investigator (NJ). Participants were enrolled by a research fellow (SJ), who also assigned participants to their treatment groups. Allocation concealment was securely maintained by storage in sealed, sequentially-numbered opaque envelopes until the interventions were assigned. Allocation was strictly maintained sequentially, all envelopes in the sequence being used. The allocated groups were intended to be analysed for clinical outcomes on an intention to treat (ITT) basis, as well as being analysed by the a priori determined methods described.

It was not possible to blind participants to treatment allocation since the treatment involved immediate HSG procedure and the control involved delayed HSG. There was also no blinding of the executor of the assignment, the clinician performing the lipiodol procedure, nor of the assessor of clinical outcomes at follow-up. However the assessor of endometrial gene expression analyses was blinded to the origin of the endometrial samples in terms of treatment allocation (see below).

The protocol dictated that the immediate lipiodol group underwent a lipiodol procedure on the cycle of randomisation followed by an endometrial sampling at day 5 post-ovulation (day 6 after the peak of the LH surge, determined by daily serum LH estimations); the delayed lipiodol group had an endometrial sampling at day 5 post-ovulation on the cycle of randomisation then, after three completed menstrual cycles following randomisation, they underwent a lipiodol procedure followed by an endometrial sampling at day 5 after ovulation (Fig. 1). Lipiodol procedures were performed as previously described (Johnson et al., 2004) after menses, on or before day 12 of the cycle, by one of the investigators $(\mathrm{NJ}$ or $\mathrm{WH})$, in a radiology facility under fluoroscopic screening at Auckland Radiology Group, using the Leech-Wilkinson cannula as the delivery system. The endometrial sampling procedures were performed by pipelle sampling - using a repeated sampling technique up to three passes until it was deemed that sufficient tissue had been retrieved - by one of the investigators (NJ or SJ). After collection, the endometrial samples were divided into two approximately equal parts. The first part was placed into RNAlater (Life Technologies, NY, USA) and was subsequently used to extract RNA needed for microarray and reverse transcriptase quantitative polymerase chain reaction (RT-qPCR) analysis. The second part was prepared for immunohistochemical analyses and these results will be presented elsewhere (Baidya et al., 2009).

The primary clinical outcome was clinical pregnancy (positive pregnancy test with positive intrauterine gestation sac on ultrasound), with last menstrual period (LMP) commencing within 9 months following randomisation, that led to live birth. Clinical data were collected from study participants by a telephone consultation with a member of the research team (SJ). Other outcomes were the upor down-regulated gene expression based on microarray studies of endometrial tissue.

For microarray analysis of the endometrial tissue, total RNA from the endometrial biopsies were isolated using TRIzol (Life Technologies) and purified using RNA affinity mini columns (manufacturer's protocol: Qiagen, Hilden, Germany). RNA yields and purity were determined using the NanoDrop spectrophotometer (NanoDrop Technologies Inc., DE, USA) and RNA integrity was assessed using the Agilent 2100 Bioanalyser (Agilent Technologies Inc., CA, USA). Total RNA ( $5 \mu \mathrm{g}$ ) was labelled using the MessageAmp ${ }^{\text {tw }}$ Premier RNA Amplification Kit (manufacturer's instructions; Life Technologies), and hybridised to Affymetrix Human Genome U133 Plus 2.0 gene chips (Affymetrix, Santa Clara, CA, USA) by the Centre of Proteomics and Genomics (The University of Auckland, NZ) according to manufacturer's instructions.

Bioinformatic analysis was carried out using the ' $\mathrm{R}$ ' statistical framework (R Core Team, 2013). Raw microarray "cel" files were background corrected, summarised, normalised and log2-transformed using the Robust Multichip Averaging (RMA) algorithm (Irizarry et al., 2003), as implemented in the R "affy" package (Gautier et al., 2004). Independent Component Analysis (ICA), which is a machine learning method useful for separating gene expression changes associated with different experimental parameters, was performed using Matlab software as we have previously described (Saidi et al., 2004). Paired statistical analysis of differential abundance between groups was performed on the log2-transformed data using the "linear models for microarray data” (LIMMA) package (Smyth et al., 2005). Given the small number of replicates available, a consistency of change filtering step was also included, where genes were prioritised if their expression was 1.5-fold up- or down-regulated in the same direction 
following lipiodol treatment in at least 3 of the 4 women's 'before' and 'after' endometrial samples. Lists of differentially expressed probe sets were tested for enrichment of particular functional categories using Ingenuity Pathways Analysis (IPA; Ingenuity Systems, http:// www.ingenuity.com, Redwood, CA), GeneSetDB (Araki et al., 2012) and GATHER (Chang and Nevins, 2006) web applications, which incorporated functional information from the Gene Ontology (GO) database (Ashburner et al., 2000).

RT-qPCR was used to validate a subset of the microarray study findings. Eight RNAs were chosen for RT-qPCR validation. These RNAs were selected on the basis of passing the criteria for differential expression described above, as well as encoding proteins that are associated with cell growth, cell signalling and interaction, cell death, immune response, metabolic disease or reproductive system disease (biological plausibility), or being previously reported in the literature to be regulated in infertility-related endometrial pathologies (prior evidence of gene regulation). Total RNA was DNase treated (manufacturer's protocol; Deoxyribonuclease I Amplification Grade Kit, Life Technologies), reverse transcribed to cDNA (manufacturer's protocol;Superscript III FirstStrandSynthesis System for RT-PCR, Life Technologies) and diluted four-fold with sterile water. The efficiency of the reverse transcription was assessed by PCR amplification of an ACTB (forward 5'-3': CCTCGCCTTTGCCGATCC; reverse 5'-3': GGATCTTCATGAGGTAGTCAGTC) fragment from the cDNA using standard PCR cycling conditions (annealing temperature of $58^{\circ} \mathrm{C}$ ). Using RT-qPCR (manufacturer's protocol; Platinum SYBR Green qPCR SuperMix-UDG with ROX, Life Technologies), cDNA samples were assayed in triplicate for the expression of the eight selected RNAs and for two housekeeping RNAs: PPIA (a member of the peptidyl-propyl isomerase family) and RPLPO (a component of the $60 \mathrm{~S}$ subunit of ribosomes), and data were analysed using the $\Delta \Delta \mathrm{C}_{\mathrm{T}}$ method, normalising against the geometric mean of the two housekeeping RNAs (Schmittgen and Livak, 2008). See Supplementary Table 1 for primer sequences.

\section{RESULTS}

Participant flow and clinical outcomes

Twelve women were recruited and randomised between August 2005 and July 2006. Follow-up continued for nine months from randomisation to April 2007 and outcomes of all pregnancies were ascertained in March 2008. Figure 1 shows the flow of participants through the study. Eleven out of the 12 women had successful endometrial sampling procedures. Clinical outcomes are clarified in Table 1. Two women receiving lipiodol in the immediate treatment group had spontaneous pregnancies within 6 months (one woman became pregnant within 3 months post-lipiodol but miscarried; the other woman had a pregnancy within 6 months post-lipiodol that eventuated in live birth); a further woman became pregnant through in vitro fertilisation (IVF) treatment between 6 and 9 months postlipiodol (and this woman was the only participant to undergo further fertility treatment during the follow-up phase of the study). In the delayed treatment group, two women became pregnant in the 3 months prior to planned lipiodol treatment (one woman miscarried then received lipiodol at the planned time 3 months postrandomisation; the other woman had an ongoing pregnancy that eventuated in live birth thus did not receive lipiodol); two further women became pregnant following lipiodol (both within 3 months, although it was not possible to obtain a pipelle endometrial sample from one of these women); the woman who miscarried prior to lipiodol again became pregnant within 3 months post-lipiodol but miscarried again. There were no reported adverse events from the lipiodol procedures or the endometrial sampling procedures.

\section{Microarray analyses}

To identify possible biological mechanisms by which lipiodol bathing may alter endometrial receptivity, microarray analysis was undertaken on endometrial tissue from four of the women in the delayed treatment group, comparing pre- and post-lipiodol treatment gene expression profiles (two other women randomised to

Fig. 1. Flow of participants through the trial.

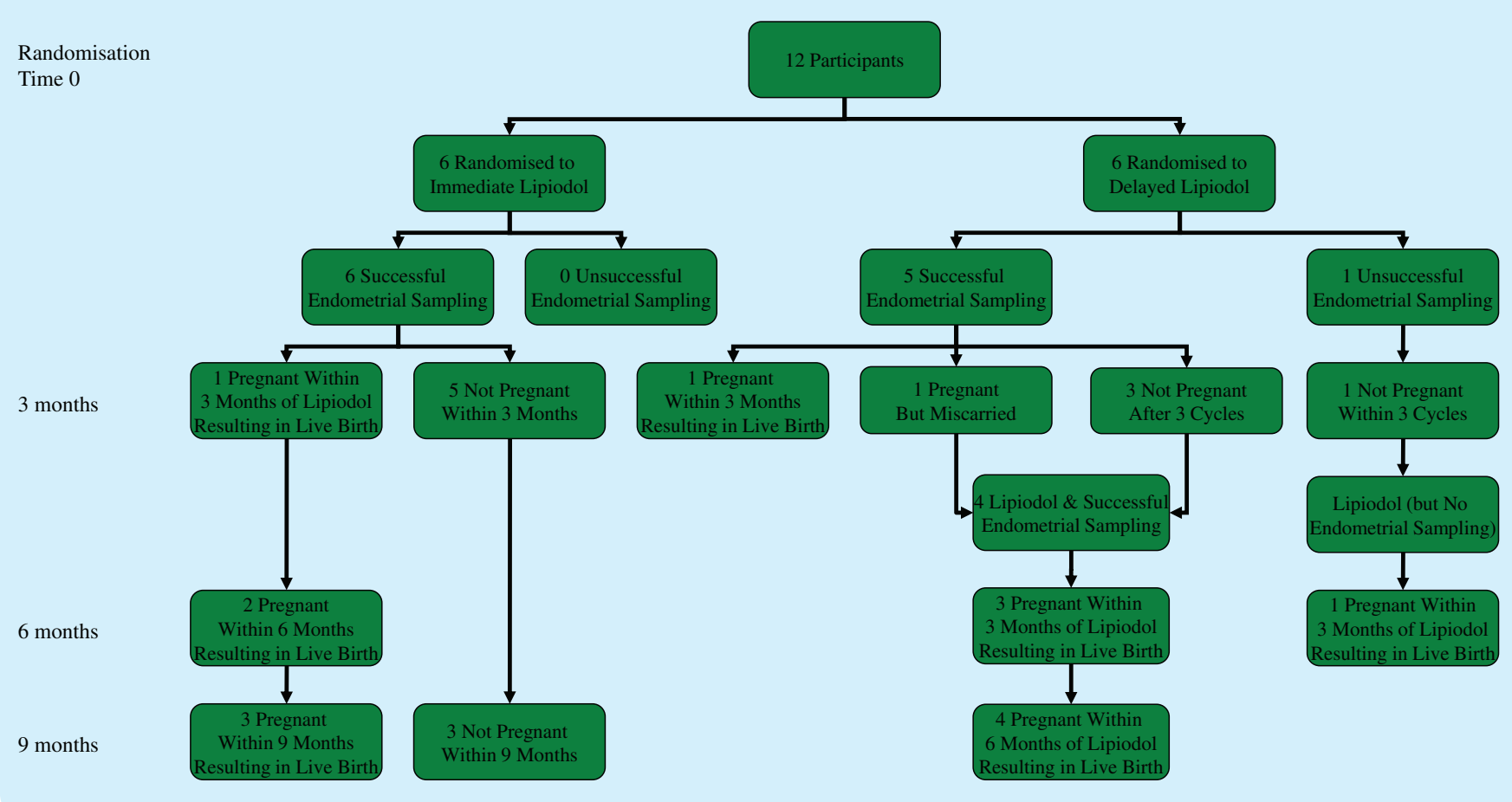


Table 1. Clinical outcomes for study population.

\begin{tabular}{lllll}
\hline Immediate lipiodol (IL) group & & & \\
\hline Patient & Diagnosis & Pregnant at 3 months? & Pregnant at 6 months? & Samples available? \\
\hline IL 1 & Prev lipiodol success & No & No & Yes \\
IL 2 & Endometriosis & No & Yes (IVF) & Yes \\
IL 3 & Endometriosis & No & No & Yes \\
IL 4 & Endometriosis & No & Yes (spontaneous) & Yes \\
IL 5 & Endometriosis & Yes (spontaneous) & & Yes \\
IL 6 & Endometriosis & No & Yes (IVF) & Yes \\
\hline Delayed lipiodol (DL) group & & & \\
\hline Patient & Diagnosis & Pregnant at 3 months (pre-lipiodol)? & Pregnant at 6 months (post-lipiodol)? & Samples available? \\
\hline DL 1 & Endometriosis & Yes (spontaneous, but miscarried) & No & Yes, both \\
DL 2 & Endometriosis & No & Yes (spontaneous) & Yes, both \\
DL 3 & Endometriosis & No & Yes (by IUI, converted to IUI from IVF) & Yes, both \\
DL 4 & Endometriosis & No & No & Yes, both \\
DL 5 & Endometriosis & No & Yes & No \\
DL 6 & Endometriosis & No & No & Yes, both \\
\hline
\end{tabular}

delayed treatment were unable to provide before and after samples owing to pregnancy in one case and unsuccessful sampling in the other case). Data processing by ICA identified strong patient-specific gene expression patterns that were robust from the first to the second endometrial sampling in those women who successfully underwent repeat endometrial sampling, as well as samples that had lower RNA yield than others (Fig. 2). However the ICA was not sensitive enough to visualise any systematic effects associated with lipiodol bathing or subsequent pregnancy (Fig. 2). Nevertheless, paired statistical analysis (using the LIMMA method with consistency of change filtering) identified 37 Affymetrix probe sets (corresponding to 33 distinct mRNAs) that appeared to be regulated in endometrial expression following lipiodol bathing. For four mRNAs there were mutliple concordantly regulated probe sets (ATAD2, SORD, NRP1 and $O A F$ ), adding confidence to the results for these mRNAs. Analyses using the GeneSetDB and GATHER web tools showed that the differentially expressed mRNAs were significantly enriched for mRNAs associated with inflammation (e.g. GATHER Bayes factor = 7, $\mathrm{p} \leq 0.0001$ for gene set GO:0006954 'inflammatory response'). These included the inflammation-associated mRNAs: AIF1, CCR1, CXCL10, SERPINA3 and SPP1 (osteopontin). Osteopontin (SPP1) was the only gene transcript regulated in all four women. These mRNAs are described in Supplementary Table 2 and in Fig. 3.

\section{RT-qPCR analyses for mRNA}

As proof of principle a small subset of the differentially expressed RNAs were validated using RT-qPCR. After normalisation of the data against two housekeeping genes, RT-qPCR gave good amplification above the threshold with one distinct peak for each sample for four mRNAs (CD86, HLA-DMB, SERPINA3 and SPHK1). The other four RNAs (BCL2A1, CCR1, HPSE and SPP1) failed to amplify above the threshold level after 40 cycles of PCR and this was confirmed in a repeat RT-qPCR for the four genes failing to amplify above the threshold, indicating their expression was insufficient for reliable quantification by RT-qPCR in these samples using the selected primers. Hence RT-qPCR validation of the microarray results was performed for only four mRNAs (CD86, HLA-DMB, SERPINA3 and $S P H K 1)$. Of the four mRNAs selected, one of these (CD86) could not be assessed in three samples. The remainder demonstrated
Fig. 2. Hinton plot showing Independent Component Analysis (ICA) of microarray data. ICA identified gene expression patterns associated with clinical and technical parameters, but not with lipiodol flushing. Samples analysed by microarray are shown in columns, and gene expression patterns (components) are shown in rows. 'Pre' and 'Post' indicate timing of endometrial biopsy relative to lipiodol flushing. Letters A-D indicate individual patients. Yellow and red boxes indicate positive and negative component values, respectively, while the size of boxes indicates component magnitude. One component (component 1) appeared to be associated with a technical feature of the endometrial

samples, since it was associated with RNA yield from the tissues. Three components appeared to be associated with individual patients irrespective of lipiodol treatment (components 10,13 and 15 associated with patients A, B and $\mathrm{D}$, respectively). No components associated with a lipiodol bathing effect were identified.

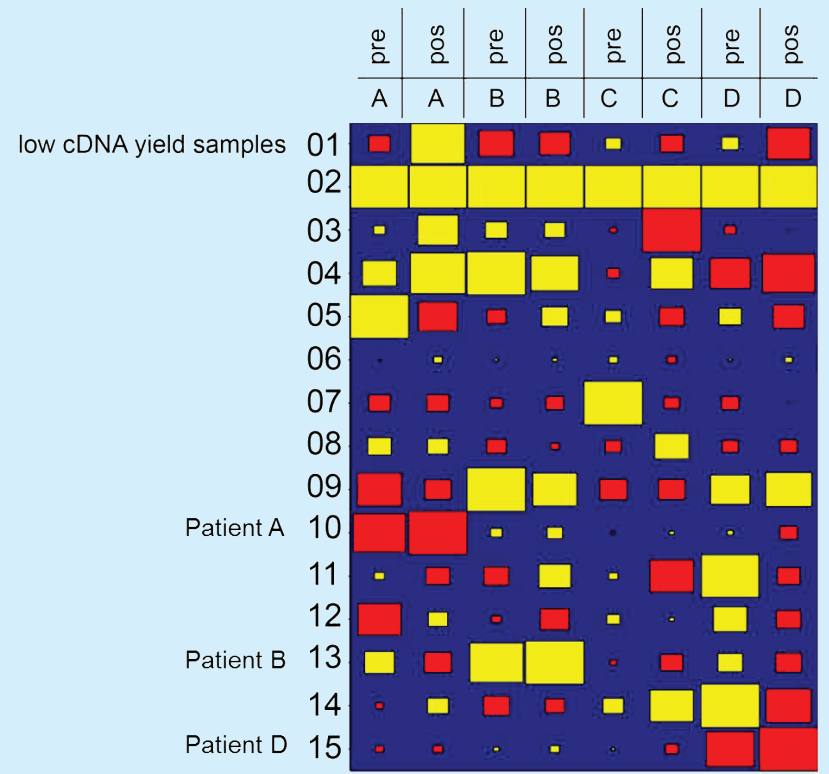


Fig. 3. Heatmap showing mRNAs identified to be differentially expressed in a paired LIMMA analysis comparing pre- and post-lipiodol treated endometrial samples. Tissue biopsies are shown in columns, with numbers indicating patients, and with 'Pr' and 'Po' indicating the biopsy was taken pre- or post lipiodol flushing, respectively. Microarray RNA expression data were transformed to standardised Z-scores then mapped to colours, with red indicating low and blue high RNA abundance (see colour key at bottom of diagram). Affymetrix probe sets IDs are shown in rows, with the official gene symbol corresponding to each probe set given as a suffix to the probe set ID. Probe sets are arranged so the probe set most significantly down-regulated after lipiodol bathing is at the top of the figure, while the probe set most significantly up-regulated after lipiodol bathing is at the bottom, with a white horizontal line separating down- and up-regulated probe sets. Red arrows indicate probe sets chosen for RT-qPCR validation.

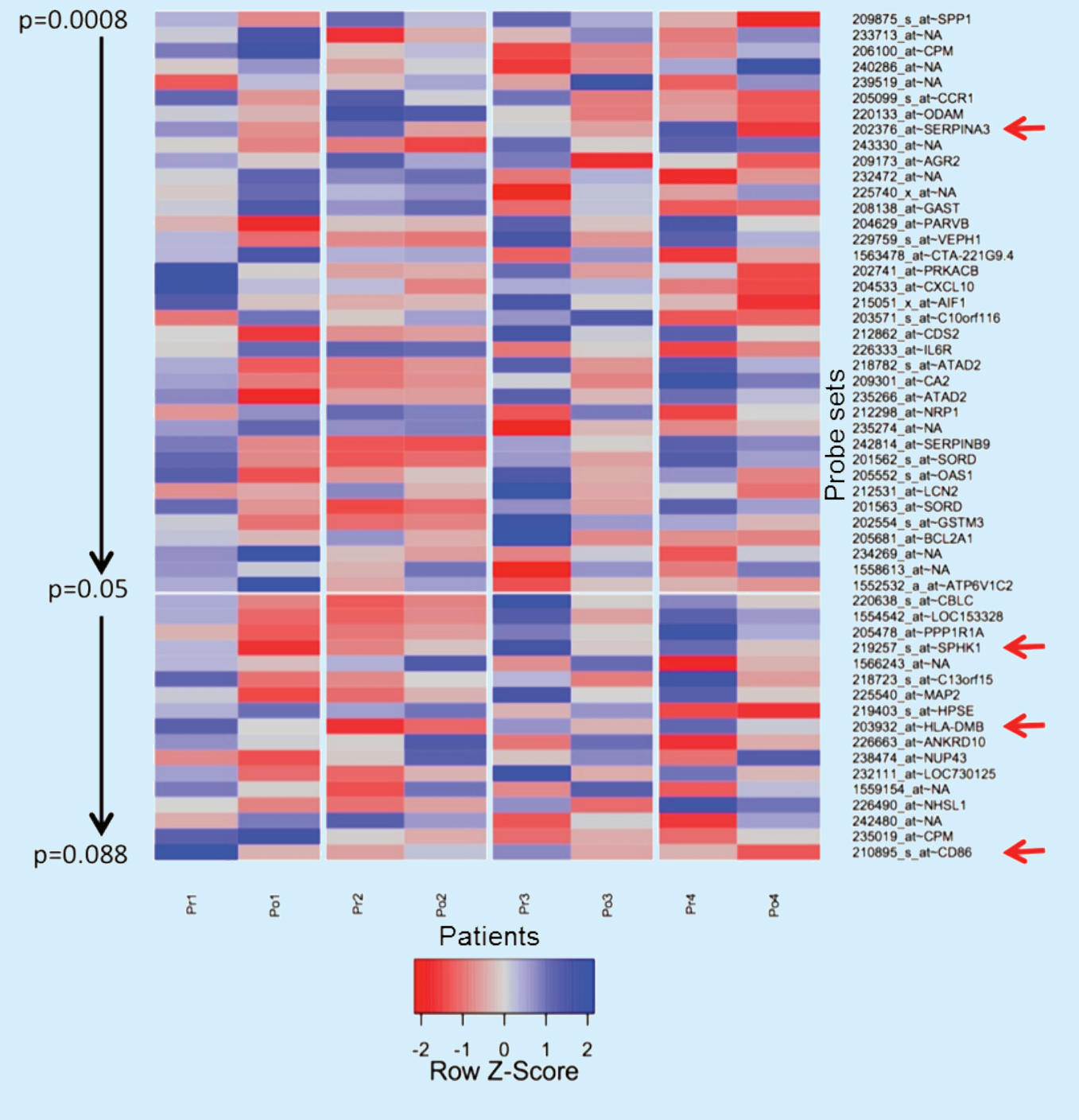

concordant regulation (with the microarray data) by lipiodol in at least three of the four women (Fig. 4).

\section{DISCUSSION}

The main finding from this study was that microarray analysis indicated 20 genes were down-regulated (reflecting 22 probe sets, but in two instances there were two probes sets for the same gene) and 13 genes up-regulated (reflecting 15 probe sets, but in two instances there were again two probes sets for the same gene) with $\mathrm{p} \leq 0.05$ and with magnitude of change $\geq 1.5$-fold in the endometrium of at least three out of four women exposed to lipiodol. Osteopontin was the only gene transcript that was regulated in the endometrium of all women exposed to lipiodol, osteopontin being down-regulated.

Of the three predominating theories for the mechanism of lipiodol fertility enhancement - that lipiodol is an effective 'flushing agent' of amorphous debris from fallopian tubes (Watson et al., 1994); that it alters peritoneal immuno-biology through an increase in regulatory T-cells apparently mediated by a change in the activation status of mature myeloid dendritic cells due to incorporation of lipiodol by these dendritic cells (Saidi et al., 2004); that it may act through a uterine bathing effect which improves endometrial receptivity (Johnson, 2005; Johnson, 2014; Izumi et al., 2017) - this study goes some way to supporting the latter uterine bathing theory. An immuno-biological effect of lipiodol is supported by the more dramatic short term effect on fertility among women with endometriosis (Johnson et al., 2004) and an endometrial effect is further supported by our murine study that showed an increase in endometrial activated dendritic cells in mice exposed to lipiodol (Johnson et al., 2005). The lipiodol uterine bathing theory is further supported in our previous study by eight spontaneous pregnancies 
Fig. 4. Comparison of RT-qPCR and microarray endometrial expression data for four women pre- and post-lipiodol bathing. For each of the four mRNAs, fold changes pre- to post-lipiodol bathing in endometrial mRNA abundance are shown for the microarray data in blue and for the RT-qPCR data in red. RT-qPCR data could not be generated for $\mathrm{CD} 86$ for three of the four paired patient samples.

SERPINA3

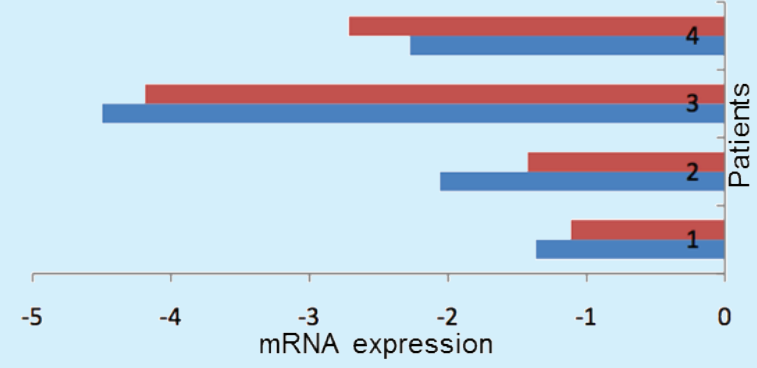

(A)

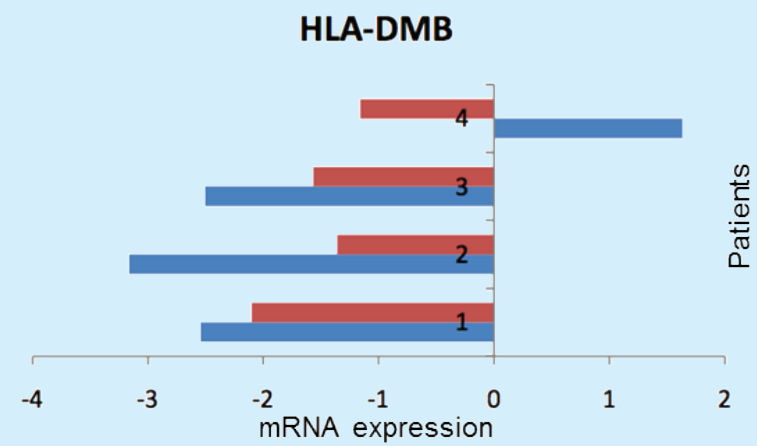

(C)
SPHK1

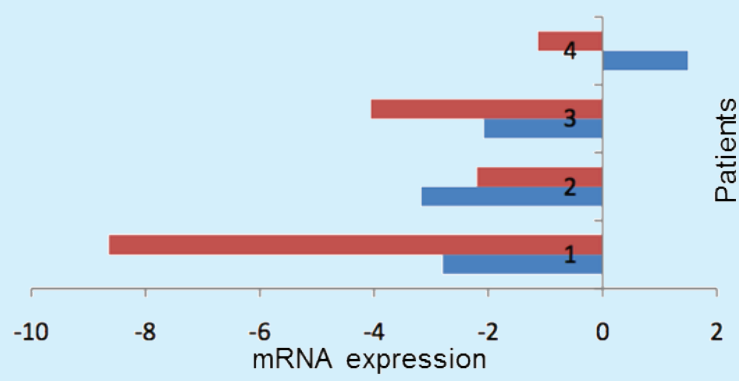

(B)

CD86

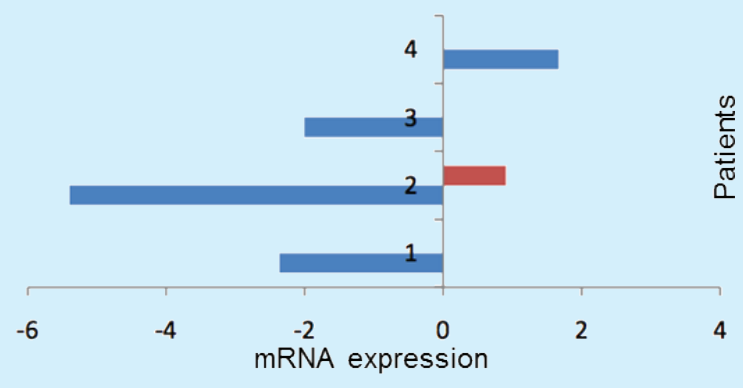

(D) amongst 17 women in whom lipiodol did not pass through either fallopian tube (although bilateral tubal patency had previously been confirmed), a pregnancy rate of $47 \%$ amongst women whose fallopian tubes remained 'unflushed' in their lipiodol procedure (Court et al., 2014), which compares favourably with the $40 \%$ pregnancy rate amongst all women receiving lipiodol (Court et al., 2014). It has been debated whether the adverse impact of endometriosis on fertility results purely from established poorer egg quality or whether the endometrium of women with endometriosis is less receptive (Pellicer et al., 2001). The eutopic endometrium of women with endometriosis has been recognised to have different expression of bio-markers associated with the implantation window compared to women who do not have endometriosis (Wei et al., 2009). In particular, women with endometriosis have been shown to overexpress endometrial osteopontin (Cho et al., 2009; Hapangama et al., 2012), a molecule whose binding with $\operatorname{av} \beta 3$ integrins is important to promote endometrial implantation receptivity and whose expression to just the right level in endometrium appears to be exacting in its impact on implantation receptivity (Johnson et al., 2003). The fact that osteopontin was the only candidate gene consistently downregulated by lipiodol in the endometrium of all four women in our study, along with the regulation of other inflammation-associated genes such as AIF1, CCR1, CXCL10 and SERPINA3, suggests a putative mechanism involving down-modulation of endometrial inflammation for improving fertility soon after lipiodol uterine bathing, which merits additional study.

There were a number of weaknesses in this study. As with any small trial, the power to detect small differences was limited, and this was likely why no clear signals appeared beyond the robust sample-to-sample signatures of individual women. Although the study was designed with the intention of comparing microarray results in women randomised to immediate lipiodol versus those not randomised to immediate lipiodol and women subsequently becoming pregnant versus those not subsequently becoming pregnant, the gene signatures of these women's endometrium were so characteristic of each individual that the differences between the participants' idiosyncratic expression profiles appeared to be much larger than the lipiodol-induced gene expression changes. Thus, although 12 participants entered the pilot randomised trial, the useful analysis could only be undertaken in one arm of the RCT the delayed treatment group. So despite the intention to make comparisons across groups and to correlate the laboratory findings with clinical outcomes, ultimately the meaningful part of the study could be considered as a prospective own-control study in a small cohort of women recruited for one treatment arm of this RCT. And even for this comparison of pre- and post-lipiodol amongst individual women, the results were based only on four women, rather than on six women in this category as was originally hoped (we had anticipated that the ability to make this comparison would be a strength of our study), since one woman in the delayed lipiodol group had a pregnancy in the first 3 months that resulted in a live birth so that only a pre-lipiodol sample and no post-lipiodol sample was available, and another woman had unsuccessful endometrial sampling procedures in whom it was not possible to obtain endometrial tissue by pipelle sampling. Further, it was disappointing that osteopontin differential expression could not be validated using RT-qPCR, however this was due to technical reasons, rather than being due to any issue with the osteopontin RT-qPCR results being 
non-concordant with the array results. It is likely that osteopontin had too low an expression to be measurable and it is recognised that RT-qPCR may sometimes be less sensitive than microarrays. Nonetheless our inability to verify the osteopontin microarray data means that a substantial cautionary note needs to be applied to our conclusions, which can, for all the reasons above, only be 'suggestive' at best.

\section{CONCLUSION}

Our study provides suggestive data to support the concept of a uterine bathing effect of lipiodol. Whether the down-regulation of endometrial inflammatory regulators, including osteopontin, might contribute to an increase in endometrial receptivity to embryo implantation merits further investigation.

\section{ACKNOWLEDGEMENTS}

We are grateful to the patients who kindly participated in this study and to the staff at Fertility Plus, Auckland, New Zealand for their assistance.

\section{CONFLICT OF INTEREST}

Neil Johnson reports a consultancy for Guerbet, the company that manufactures lipiodol, from 2016 to date.

\section{REFERENCES}

Araki H, Knapp C, Tsai P, Print C. GeneSetDB: a comprehensive meta-database, statistical and visualisation framework for gene set analysis. FEBS Open Biol. 2012;2:76-82.

Ashburner M1, Ball CA, Blake JA, Botstein D, Butler H, Cherry JM, Davis AP, Dolinski K, Dwight SS, Eppig JT, Harris MA, Hill DP, Issel-Tarver L, Kasarskis A, Lewis S, Matese JC, Richardson JE, Ringwald M, Rubin GM, Sherlock G. Gene ontology: tool for the unification of biology. The Gene Ontology Consortium. Nat Genet. 2000;25:25-9.

Baidya S, Print C, Chamley L, Shelling A, Muthukaruppan A, Johnson $\mathrm{N}$. Endometrial changes associated with lipiodol uterine bathing. In: 21 st AOCOG Combined with the RANZCOG Annual Scientific Meeting, Sky City, Auckland, 26-30 March 2009; p. 46.

Chang JT, Nevins JR. GATHER: a systems approach to interpreting genomic signatures. Bioinformatics. 2006;22:2926-33.

Cho S, Ahn YS, Choi YS, Choi YS, Seo SK, Nam A, Kim HY, Kim JH, Park KH, Cho DJ, Lee BS. Endometrial osteopontin mRNA expression and plasma osteopontin levels are increased in patients with endometriosis. Am J Reprod Immunol. 2009;61:286-93.

Court KA, Dare AJ, Weston-Webb M, Hadden WE, Sim RG, Johnson NP. Establishment of lipiodol as a fertility treatment - prospective study of the complete innovative treatment data set. Aust N Z J Obstet Gynaecol. 2014;54:13-9.

Dreyer K, van Rijswijk J, Mijatovic V, Goddijn M, Verhoeve HR, van Rooij IAJ, Hoek A, Bourdrez P, Nap AW, Rijnsaardt-Lukassen HGM, Timmerman CCM, Kaplan M, Hooker AB, Gijsen AP, van Golde R, van Heteren CF, Sluijmer AV, de Bruin JP, Smeenk JMJ, de Boer JAM, Scheenjes E, Duijn AEJ, Mozes A, Pelinck MJ, Traas MAF, van Hooff MHA, van Unnik GA, de Koning CH, van Geloven N, Twisk JWR, Hompes PGA, Mol BWJ. Oil-based or water-based contrast for hysterosalpingography in infertile women. N Engl J Med. 2017;376:2043-52.

Gautier L, Cope L, Bolstad BM, Irizarry RA. Affy - analysis of Affymetrix GeneChip data at the probe level. Bioinformatics. 2004;20:307-15.
Hapangama DK, Raju RS, Valentijn AJ, Barraclough D, Hart A, Turner MA, Platt-Higgins A, Barraclough R, Rudland PS. Aberrant expression of metastasis-inducing proteins in ectopic and matched eutopic endometrium of women with endometriosis: implications for the pathogenesis of endometriosis. Hum Reprod 2012;27: 394-407.

Irizarry RA, Bolstad BM, Collin F, Cope LM, Hobbs B, Speed TP. Summaries of Affymetrix GeneChip probe level data. Nucleic Acids Res. 2003;31:e15.

Izumi G, Koga K, Takamura M, Bo W, Nagai M, Miyashita M, Harada M, Hirata T, Hirota Y, Yoshino O, Fujii T, Osuga Y. Oil-soluble contrast medium (OSCM) for hysterosalpingography modulates dendritic cell and regulatory $\mathrm{T}$ cell profiles in the peritoneal cavity: a possible mechanism by which OSCM enhances fertility. J Immunol. 2017;198:4277-84.

Johnson GA, Burghardt RC, Bazer FW, Spencer TE. Osteopontin: roles in implantation and placentation. Biol Reprod. 2003;69: 1458-71.

Johnson NP. A review of the use of lipiodol flushing for unexplained infertility. Treat Endocrinol. 2005;4:233-43.

Johnson NP. In: Lipiodol Fertility Enhancement in Unexplained and Endometriosis-Related Infertility. Thesis, University of Auckland, 2007, Chap. 12, p. 160-6.

Johnson NP. A review of lipiodol treatment for infertility - an innovative treatment for endometriosis-related infertility? Aust N Z J Obstet Gynaecol. 2014;54:9-12.

Johnson NP, Bhattu S, Wagner A, Blake DA, Chamley LW. Lipiodol alters murine uterine dendritic cell populations: a potential mechanism for the fertility enhancing effect of lipiodol. Fertil Steril. 2005;83:1814-21.

Johnson NP, Farquhar CM, Hadden WE, Suckling J, Yu Y, Sadler L. The FLUSH Trial - Flushing with lipiodol for unexplained (and endometriosis-related) subfertility by hysterosalpingography: a randomised trial. Hum Reprod. 2004;19:2043-51.

Mohiyiddeen L, Hardiman A, Fitzgerald C, Hughes E, Mol BW, Johnson N, Watson A. Tubal flushing for subfertility. Cochrane Database Syst Rev. 2015;5:CD003718.

Pellicer A, Navarro J, Bosch E, Garrido N, Garcia-Velasco JA, Remohí J, Simón C. Endometrial quality in infertile women with endometriosis. Ann N Y Acad Sci. 2001;943:122-30.

R Core Team. R: A language and environment for statistical computing. R Foundation for Statistical Computing. URL http://www.Rproject.org/, 2013; Vienna, Austria.

Saidi SA, Holland CM, Kreil DP, MacKay DJC, Charnock-Jones DS, Print CG, Smith SK. Independent Component Analysis is a valid model for microarray analysis in endometrial cancer. Oncogene. 2004;23:6677-83.

Schmittgen TD, Livak KJ. Analyzing real-time PCR data by the comparative C(T) method. Nat Protoc. 2008;3:1101-8.

Smyth GK. Limma: linear models for microarray data. In: Gentleman R, Carey V, Dudoit S, Irizarry R, Huber W (editors). Bioinformatics and Computational Biology Solutions Using R and Bioconductor. New York: Springer-Verlag; 2005: p. 397-420.

Watson A, Vandekerckhove P, Lilford R, et al. A meta-analysis of the therapeutic role of oil soluble contrast media at hysterosalpingography: a surprising result? Fertil Steril. 1994;61:470-7.

Wei Q, St Clair JB, Fu T, Stratton P, Nieman LK. Reduced expression of biomarkers associated with the implantation window in women with endometriosis. Fertil Steril. 2009;91:1686-91. 
Supplementary Table 1. RT-qPCR primer sequences.

\begin{tabular}{|c|c|c|c|}
\hline Gene symbol & Gene description & Primer orientation & $5^{\prime}$ to $3^{\prime}$ sequence \\
\hline \multirow[t]{2}{*}{ BCL2Al } & \multirow[t]{2}{*}{ BCL2-related protein A1 } & Forward & TTTTGTTGCGGAGTTCATAATGA \\
\hline & & Reverse & ATTTTCCCAGCCTCCGTTTT \\
\hline \multirow[t]{2}{*}{ CD86 } & \multirow[t]{2}{*}{ CD86 molecule } & Forward & CATTCCCTGATGTTACGAGCAA \\
\hline & & Reverse & GCCGCGTCTTGTCAGTTTC \\
\hline \multirow[t]{2}{*}{ HLADMB } & \multirow[t]{2}{*}{ Major histocompatibility complex class II, DM beta } & Forward & TGGTTTCTCCATCCAATTCTTTG \\
\hline & & Reverse & GGTCCCCCAAGTTGCTAAGTT \\
\hline \multirow[t]{2}{*}{ PPIA } & \multirow[t]{2}{*}{ Peptidylprolyl isomerase A } & Forward & GGGTTCCTGCTTTCACAGAATT \\
\hline & & Reverse & GGACCCGTATGCTTTAGGATGA \\
\hline \multirow[t]{2}{*}{$R P L P O$} & \multirow[t]{2}{*}{ Ribosomal protein, large, P0 } & Forward & ATGGGCAAGAACACCATGATG \\
\hline & & Reverse & CCTCCTTGGTGAACACAAAGC \\
\hline \multirow[t]{2}{*}{ SERPINA3 } & \multirow{2}{*}{$\begin{array}{l}\text { Serpin peptidase inhibitor, clade A (alpha-1 antiproteinase, } \\
\text { antitrypsin), member } 3\end{array}$} & Forward & CAGGCCCTTCCTGATGATCA \\
\hline & & Reverse & GGATTGGTGACTTTGCTCATGA \\
\hline \multirow[t]{2}{*}{ SPP1 } & \multirow[t]{2}{*}{ Secreted phosphoprotein 1} & Forward & AGATATGCTGGTTGTAGACCCCAA \\
\hline & & Reverse & TGCACTATCTAATTCATGAGAAATACGA \\
\hline
\end{tabular}

Supplementary Table 2. List of mRNAs in endometrium found to be differentially expressed following lipiodol treatment.

Along with mean fold change across the four patients and paired LIMMA p value, this list includes mRNAs that pass both of the following criteria: (i) Paired LIMMA p value of less than 0.05 and (ii) more than 1.5 fold differential expression between the pre- and post-lipiodol treatment biopsies in at least 3 of the 4 patients.

\begin{tabular}{|c|c|c|c|c|c|c|}
\hline Probe Set ID & Gene ID & Description & $\begin{array}{l}\text { Expression fold change } \\
\text { in endometrium }\end{array}$ & p Value & $\begin{array}{l}\text { Patients in which RNA } \\
\text { regulated } \geq+1.5\end{array}$ & $\begin{array}{l}\text { Patients in which RNA } \\
\text { regulated } \leq-1.5\end{array}$ \\
\hline 233713_at & OAF & OAF homolog & 1.58 & 0.00094 & 3 & 0 \\
\hline 206100_at & CPM & carboxypeptidase M & 1.82 & 0.00178 & 3 & 0 \\
\hline 240286_at & GPM6B & glycoprotein M6B & 1.59 & 0.00235 & 3 & 0 \\
\hline 239519_at & NRP1 & neuropilin 1 & 1.8 & 0.00249 & 3 & 0 \\
\hline 232472_at & FNDC3B & fibronectin type III domain containing 3B & 1.43 & 0.01000 & 3 & 0 \\
\hline 225740_x_at & MDM4 & Mdm4 p53 binding protein homolog & 1.59 & 0.01023 & 3 & 0 \\
\hline 208138_at & GAST & gastrin & 2.08 & 0.01339 & 3 & 0 \\
\hline 1563478_at & CTA-221G9.4 & KIAA1671 protein & 1.47 & 0.01608 & 3 & 0 \\
\hline 203571_s_at & C10orf116 & chromosome 10 open reading frame 116 & 1.7 & 0.02238 & 3 & 0 \\
\hline 226333_at & IL6R & interleukin 6 receptor & 1.61 & 0.02724 & 3 & 0 \\
\hline 212298 _at & NRP1 & neuropilin 1 & 1.5 & 0.02991 & 3 & 0 \\
\hline 235274_at & NA & unknown & 1.91 & 0.03073 & 3 & 0 \\
\hline 234269_at & NA & unknown & 1.44 & 0.04497 & 3 & 0 \\
\hline 1558613_at & OAF & OAF homolog & 1.56 & 0.04683 & 3 & 0 \\
\hline 1552532_a_at & ATP6V1C2 & ATPase, $\mathrm{H}+$ transporting, lysosomal $42 \mathrm{kDa}$, V1 subunit $\mathrm{C} 2$ & 1.44 & 0.04729 & 3 & 0 \\
\hline 209875_s_at & SPP1 & secreted phosphoprotein 1 (osteopontin) & -1.98 & 0.00076 & 0 & 4 \\
\hline 205099_s_at & CCR1 & chemokine (C-C motif) receptor 1 & -1.6 & 0.00286 & 0 & 3 \\
\hline 220133_at & ODAM & odontogenic, ameloblast asssociated & -1.86 & 0.00295 & 0 & 3 \\
\hline 202376_at & SERPINA3 & serpin peptidase inhibitor, clade A member 3 & -2.32 & 0.00488 & 0 & 3 \\
\hline 243330_at & LRRTM1 & leucine rich repeat transmembrane neuronal 1 & -1.6 & 0.00715 & 0 & 3 \\
\hline 209173_at & AGR2 & anterior gradient homolog 2 & -2.05 & 0.00896 & 0 & 3 \\
\hline 204629_at & PARVB & parvin, beta & -1.41 & 0.01514 & 0 & 3 \\
\hline 229759_s_at & VEPH1 & ventricular zone expressed PH domain homolog 1 & -1.76 & 0.01548 & 0 & 3 \\
\hline 202741_at & PRKACB & protein kinase, cAMP-dependent, catalytic, beta & -1.49 & 0.01762 & 0 & 3 \\
\hline 204533_at & CXCL10 & chemokine (C-X-C motif) ligand 10 & -1.94 & 0.01850 & 0 & 3 \\
\hline 215051_x_at & AIF1 & allograft inflammatory factor 1 & -1.5 & 0.01966 & 0 & 3 \\
\hline 212862_at & CDS2 & CDP-diacylglycerol synthase (phosphatidate cytidylyltransferase) 2 & -1.41 & 0.02305 & 0 & 3 \\
\hline 218782_s_at & ATAD2 & ATPase family, AAA domain containing 2 & -1.98 & 0.02766 & 0 & 3 \\
\hline 209301_at & CA2 & carbonic anhydrase II & -2.49 & 0.02770 & 0 & 3 \\
\hline 235266_at & ATAD2 & ATPase family, AAA domain containing 2 & -1.97 & 0.02796 & 0 & 3 \\
\hline 242814_at & SERPINB9 & serpin peptidase inhibitor, clade B (ovalbumin), member 9 & -1.9 & 0.03120 & 0 & 3 \\
\hline 201562_s_at & SORD & sorbitol dehydrogenase & -2.44 & 0.03170 & 0 & 3 \\
\hline 205552_s_at & OAS1 & $2^{\prime}, 5^{\prime}$-oligoadenylate synthetase $1,40 / 46 \mathrm{kDa}$ & -1.61 & 0.03450 & 0 & 3 \\
\hline 212531_at & LCN2 & lipocalin 2 & -2.38 & 0.03955 & 0 & 3 \\
\hline 201563_at & SORD & sorbitol dehydrogenase & -2.35 & 0.04132 & 0 & 3 \\
\hline 202554_s_at & GSTM3 & glutathione S-transferase M3 (brain) & -1.51 & 0.04249 & 0 & 3 \\
\hline 205681_at & BCL2A1 & BCL2-related protein A1 & -3.73 & 0.04407 & 0 & 3 \\
\hline
\end{tabular}

